\title{
Atropine therapy in acute anticholinesterase (Organophosphorus / carbamate) poisoning; adherence to current guidelines
}

\author{
Jayasinghe $\mathrm{SS}^{1,2,3}$, Fernando $\mathrm{A}^{1}$, Pathirana $\mathrm{KD}^{3}$, K Kishanthi Gunasinghe ${ }^{2}$ \\ ${ }^{\prime}$ Department of Pharmacology, Faculty of Medicine, University of Ruhuna, Galle. \\ ${ }^{2}$ South Asian Clinical Toxicology Research Collaboration, Faculty of Medicine, University of Peradeniya. \\ ${ }^{3}$ Clinical Neuroscience Centre, Department of Medicine, Faculty of Medicine, University of Ruhuna, Galle.
}

e-mail address of the corresponding author,Dr. S.S. Jayasinghe: sudheerasj@yahoo.com

\begin{abstract}
Purpose: Atropine is a life saving drug in acute anticholinesterase poisoning. Proper atropinization is associated with better clinical outcome. Hence we aimed to look at the adherence to existing guidelines on atropine therapy in anticholinesterase poisoning.
\end{abstract}

Method: A cross-sectional study at two tertiary care hospitals was carried out by using a semi-structured questionnaire. Association between atropine toxicity and death were analyzed by Chi-squared test.

Results: Among the 144 patients with anticholinesterase poisoning (105 organophosphorus, 39 carbamate) 110 were males. Mean (SD) age was 37 (16) years. In 52 patients, atropine was started at peripheral hospitals where as atropine was commenced in 89 of the patients at the collaborating hospitals. Three patients were not treated with atropine. Fourteen (16\%) patients did not have cholinergic features at the time of commencing atropine. Features of atropine toxicity developed in $122(90 \%)$ patients. Twenty-four (17\%) patients died in spite of the treatment. Atropine toxicity was associated with the occurrence of deaths; $p<0.05$.

Conclusion: The occurrence of death due to anticholinesterase poisoning was high when compared to national figure which was $12 \%$; attention should be focused on identifying the causative factors of the high death rate. Association between atropine toxicity and death were statistically significant.

\section{Introduction}

Acute pesticide poisoning is a major health problem in many developing countries ${ }^{1}$. Suicide rates in Sri Lanka increased by 700\% between 1960 and 1997 and this was entirely due to an increase in deaths by poisoning ${ }^{2}$.

During 2003, pesticide poisoning accounted for $4.5 \%$ of deaths and ranked the sixth leading cause of death in Sri Lanka. Organophosphorus (OP) and carbamate insecticides were the commonest groups of chemicals involved in poisoning, accounting for almost $54 \%$ of the pesticide poisonings ${ }^{3}$.

According to the Annual Health Statistics 2007, there were 64,282 cases of poisoning reported in Sri Lanka.13,079 (20\%) were due to OP or carbamate poisoning. Of the total of 1,561 deaths due to poisonings, $853(55 \%)$ were due to OP or carbamate.
The prevalence of deaths as a result of anticholinesterase poisoning was $12 \%{ }^{4}$.

OP and carbamates inhibit acetylcholinesterase enzyme which degrades acetylcholine. This inhibition results in the accumulation of acetylcholine and overstimulation of acetylcholine receptors in neuro-effector junctions and in the brain. This gives rise to characteristic autonomic, central nervous system and neuromuscular features.

The principles of therapy in anticholinesterase poisoning include resuscitation of patient, oxygen, a muscarinic antagonist, fluids and acetylcholinesterase reactivator (an Oxime-only in OP poisoning) $)^{5,6}$. The role of oximes is not completely clear $^{5,6}$. Therefore, atropine is the mainstay of therapy ${ }^{5,6}$. Atropine blocks muscarinic 
receptors limiting neuro-effector transmission by excessive acetylcholine.

We aimed to look at the adherence to existing guidelines on atropine therapy in anticholinesterase poisoning by the ward staff.

Current practice of atropine therapy was compared against the guidelines published by the National Poisons Information Center, National Hospital of Sri Lanka, Colombo?.

The important features of anticholinesterase poisoning are miosis, bronchospasm / bronchorrhoea, bradycardia, hypotension and excessive sweating ${ }^{6,7}$. Therefore, the above features of the cholinergic syndrome should be considered when taking a decision whether to commence atropine therapy or not. Thereafter, the above features should also be monitored closely to check whether atropine therapy is adequate or not.

It is recommended to start atropine if patient develops cholinergic features ${ }^{6,7}$. If none of the signs are present, patient does not require atropine but careful observation is required to look for the development of cholinergic signs ${ }^{6,7}$. This is because signs may be delayed due to a pro-poison getting converted to an active form and being released into the blood later.

The aim of atropine therapy is to reverse the cholinergic features and improve cardiac and respiratory function as quickly as possible. A loading dose of intravenous atropine, $1.8-3 \mathrm{mg}$ (into a fast-flowing infusion) should be given. Atropine takes only a few minutes to act. Therefore, if a consistent improvement in the cholinergic features does not occur within 3-5 minutes after the initial loading dose, the recommendation is to double the dose, and continue to double the dose until a response is obtained ${ }^{6,7}$.

Severely poisoned patients may require hundreds of milligrams of atropine, hence simple repetition of the initial dose should not be done ${ }^{6}$.

Once atropinised (Table 1), blood atropine concentration should be kept in the therapeutic range. To achieve this, $10-20 \%$ of the total amount of atropine required to load the patient has to be given every hour as an intravenous infusion ${ }^{6,7}$. The maintenance dose should be titrated against symptoms.

\section{Table 1 - Features used to assess atropine adequacy}

Clear chest on auscultation with no wheeze

Heart rate between $80-100$ beats/min

Pupils no longer pinpoint

Systolic blood pressure $>80 \mathrm{mmHg}$

Dry axillae

After initial stabilization every patient should be assessed to see whether the atropine infusion rate is adequate or not. The infusion rate should be sufficient and there should be no signs of atropine toxicity ${ }^{6}$.

\section{Materials and methods}

A cross-sectional study was carried out from June 2008 - May 2009 at the Teaching Hospital Galle and the General Hospital, Matara. Patients admitted to the collaborating hospitals due to anticholinesterase poisoning were recruited for the study after obtaining informed written consent from the patient or a close relative available at the time of admission.

We looked into whether cholinergic features were considered to commence atropine therapy and whether patients developed atropine toxicity (Table 2) during the maintenance therapy.

\section{Table 2 - Features of atropine toxicity}

\begin{tabular}{l}
\hline Confusion \\
Pyrexia \\
Urine retention \\
Bowel ileus \\
Hypertension \\
Tachycardia \\
\hline
\end{tabular}

Although pyrexia is a feature of atropine toxicity, it was not taken into account since most patients had lower respiratory tract infection, hence pyrexia.

Data collection and monitoring of patients were done by a Clinical Research Assistant (a pre-intern medical graduate) using a semi-structured interviewer administered questionnaire. Data were obtained from monitoring sheets and case notes of the patients, clinical examination of the patients and inquiring from the patients and care givers. 


\section{Results}

Among the 144 patients (110 males) recruited for the study 105 were due to OP poisoning whereas 39 were due to carbamate poisoning. Mean (SD) age was 37 (16) and range was $14-90$ years.

Twenty four $(17 \%)$ patients were admitted to the Intensive Care Unit (ICU) during the hospital stay and 10 of them died.

In 52 patients atropine was started at peripheral hospitals before the patients were transferred. Eighty-nine of the patients were started on atropine at the collaborating hospitals. Three patients were not treated with atropine since they did not develop any cholinergic features. A single bolus dose of atropine was given to two patients; these two did not receive maintenance therapy. Two patients died while receiving the loading dose of atropine. One patient was transferred to the ICU in another hospital just after commencement of atropine. Therefore, 89 patients were considered for the analysis of adherence to the guidelines in the commencement of atropine therapy and 136 patients were considered for adherence to the maintenance therapy of atropine.

The most common cholinergic feature after anticholinesterase poisoning was miosis where as $16 / 89(18 \%)$ patients had hypotension (Figure 1).

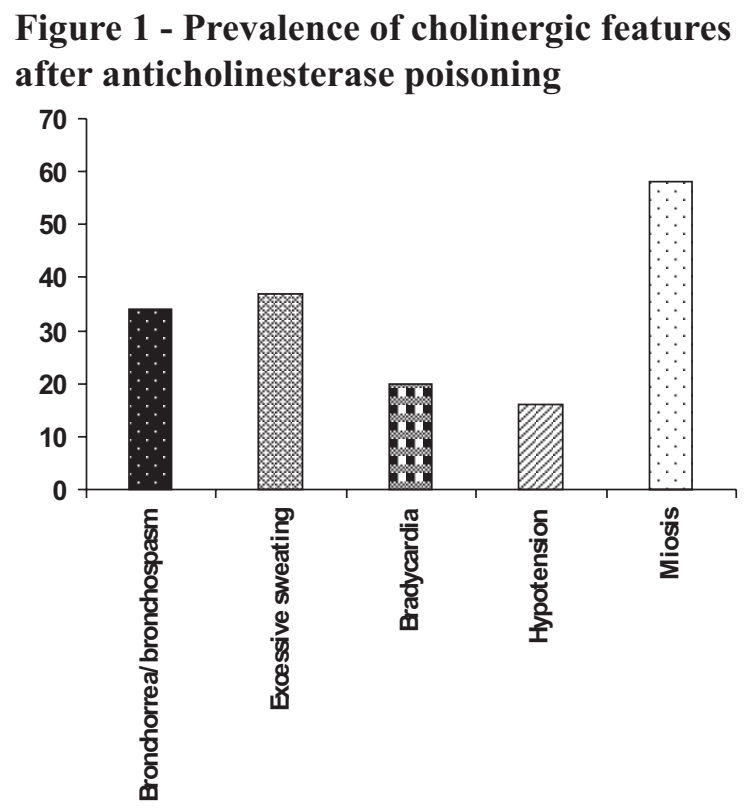

Among 89 of the patients whom atropine were started at collaborating hospitals, $14(16 \%)$ patients did not have any cholinergic features at the time of commencing atropine. Two patients were treated with a single bolus dose of atropine and one did not have cholinergic features. $23(26 \%)$ patients were put on maintenance therapy of atropine without a loading dose.

Among 136 patients who received maintenance therapy of atropine, $10(7 \%)$ patients did not have either toxic features or reappearance of cholinergic features. Four patients $(3 \%)$ had reappearance of cholinergic features. During the maintenance therapy after atropinization, $122(90 \%)$ patients in the study developed at least one feature of atropine toxicity (Figure 2). The commonest feature of atropine toxicity was tachycardia. However 118 $(87 \%)$ of patients who were on maintenance therapy of atropine developed confusion.

Figure 2 - Prevalence of features of atropine toxicity in the study group.

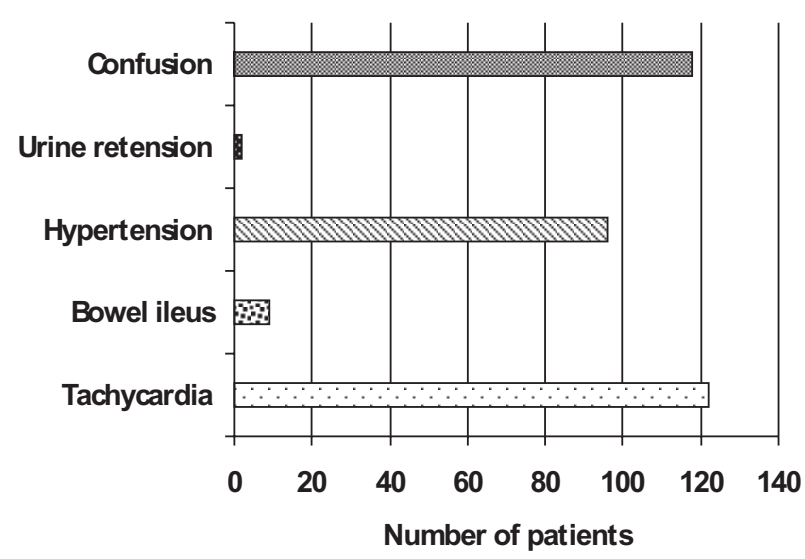

Twenty-four patients died in spite of the treatment with overall mortality of $17 \%$. Among the patients who died, 14(58\%) had features of atropine toxicity during the therapy. Four (17\%) patients had features of atropine inadequacy and six (25\%) neither had features of toxicity nor inadequacy. Association between atropine toxicity and the occurrence of death were analyzed by Chi-squared test. For the calculation all patients admitted to collaborating hospital who developed cholinergic features or who were on atropine were considered. Patients who developed atropine toxicity and who did not develop atropine toxicity during the therapy were compared against whether the final outcome was death or not. $X^{2}=4.75(p<0.05)$. 
Among 105 patients admitted due to OP poisoning $82(78 \%)$ patients were treated with pralidoxime, 17 $(16 \%)$ patients were not treated with pralidoxime and data were not available in six patients $(6 \%)$.

Among the patients who received pralidoxime complete data was available in 66 patients.

Thirty-two $(48 \%)$ patients were not treated with loading dose of pralidoxime whereas thirteen (20\%) patients did not receive maintenance dose. The median (IQR) of loading dose and maintenance dose of pralidoxime were 1.1 (1-1.5) g and 4 (3-4) g respectively.

\section{Discussion}

The prevalence of death due to anticholinesterase poisoning in the present study is high when compared with national figures. National figures may be an underestimation, but if the figures show the real picture, attention should be focused on identifying the causative factors of the high death rate.

The recommendation is not to start atropine unless the patients have cholinergic features. But there is a possibility of developing cholinergic features later. Especially with a pro-poison that converts to the active form and gets released into the blood stream later, eg. Fenthion. In such instances careful observation is required to detect the late development of cholinergic features. However, in $16 \%$ of the study group, atropine was commenced even before a single cholinergic feature appeared.

$90 \%$ of the patients developed atropine toxicity during therapy, although guidelines say that maintenance therapy with atropine after initial atropinization should be in doses adequate to control cholinergic over activity, without causing toxicity.

Atropine toxicity may lead to agitation and struggling in patients, leading to excessive heat generation and hyperthermic cardiac arrest, which could be a possible cause of death ${ }^{6}$.

When considering the infrastructure of a general medical ward in Sri Lanka, it may be difficult to administer atropine without causing toxicity. Ideally the atropine infusion should be administered via an infusion pump. General medical wards usually have at least one infusion pump but some wards do not have a single. Availability of an infusion pump for a patient with poisoning is unlikely since usually this facility is provided for patients with other medical conditions, eg. Myocardial infarction. Therefore, atropine infusions are usually administered through a drip set. Although the drip rate can be adjusted, it is more difficult to control than an infusion pump, and the atropine dose may be delivered to the body at a higher rate than optimal.

During the maintenance therapy of atropine, close monitoring is required and the dose should be titrated against the cholinergic features. Close monitoring and titration may be difficult in a busy ward especially when the staff is inadequate.

There is a risk in fine tuning an atropine infusion in the absence of close monitoring facilities. An inadequate infusion will result in a recurrence of the cholinergic syndrome that will compromise tissue perfusion and hence life.

Therefore, it may be safer to keep the patient maintained on a higher than optimal dose, but a significant association between atropine toxicity and death of patients has been shown.

High dependent unit with a close monitoring facilities and titration of atropine against symptoms may improve the outcome of the patients with anticholinesterase poisoning.

\section{Acknowledgements}

We would like to acknowledge Professor Nick Buckley, Professor Andrew Dawson, all consultants who gave permission to include their patients in the study, administrative staff and health care professionals of Teaching Hospital, Karapitiya and General Hospital, Matara, Heads and staff members of the Department of Pharmacology and the Department of Medicine, Faculty of Medicine, University of Ruhuna, Sri Lanka and Clinical Research Assistants, SACTRC.

\section{Sources of funding}

Wellcome Trust and NHMRC International Collaborative Research Grant (GR071669MA). 


\section{References}

1. Roberts DM, et al. Influence of pesticide regulation on acute poisoning deaths in Sri Lanka. Bull World Health Organ, 2003; 81(11): p789-98.

2. Van der Hoek W, F Konradsen. Risk factors for acute pesticide poisoning in Sri Lanka. Trop Med Int Health 2005; 10(6): p589-96.

3. Bulletin AH, Annual Health Bulletin 2003, Health Ministry Sri Lanka. p21-30.

4. Statistics AH, Morbidity and mortality. AH Statistics, Editor 2007, Medical Statistics Unit, p38.

5. Eddleston $M$, et al. Management of acute organophosphorus pesticide poisoning. Lancet, 2008; 371(9612): p597-607.

6. Eddleston M. Dawson A, Karalliedde L., Dissanayake W. Hettiarage A. Azher S. Buckley NA. Early management after self-poisoning with an organophosphorus or carbamate pesticide - a treatment protocol for junior doctors. Critical Care 2004 Dec; 8(6): R391-7.

7. Fernando R, Management of poisoning. Organophosphates 2007: National Poisons Information Centre, National Hospital of Sri Lanka, Colombo, 42-52. 